\title{
Multifunctional roles of the mammalian CCR4-NOT complex in physiological phenomena
}

\author{
Yo-Taro Shirai ${ }^{1}{ }^{*}$, Toru Suzuki $^{1}$, Masahiro Morita ${ }^{2,3}$, Akinori Takahashi ${ }^{1}$ and Tadashi Yamamoto ${ }^{1}$ * \\ ' Cell Signal Unit, Okinawa Institute of Science and Technology Graduate University, Onna-son, Japan \\ 2 Department of Biochemistry, McGill University, Montreal, OC, Canada \\ ${ }^{3}$ Goodman Cancer Research Centre, McGill University, Montreal, OC, Canada
}

\section{Edited by:}

Martine Anne Collart, University of

Geneva, Switzerland

\section{Reviewed by:}

Toshifumi Inada, Tohoku University Japan

Sumit Arora, University of South Alabama, USA

\section{${ }^{*}$ Correspondence:}

Yo-Taro Shirai and Tadashi Yamamoto, Cell Signal Unit, Okinawa Institute of Science and Technology Graduate University, 1919-1 Tancha Onna-son, Kunigami-gun, Okinawa 904-0495, Japan

e-mail: yotarowhite@oist.jp;

tyamamot@ims.u-tokyo.ac.jp
The carbon catabolite repression 4 (CCR4)-negative on TATA-less (NOT) complex serves as one of the major deadenylases of eukaryotes. Although it was originally identified and characterized in yeast, recent studies have revealed that the CCR4-NOT complex also exerts important functions in mammals, -including humans. However, there are some differences in the composition and functions of the CCR4-NOT complex between mammals and yeast. It is noteworthy that each subunit of the CCR4-NOT complex has unique, multifunctional roles and is responsible for various physiological phenomena. This heterogeneity and versatility of the CCR4-NOT complex makes an overall understanding of this complex difficult. Here, we describe the functions of each subunit of the mammalian CCR4-NOT complex and discuss the molecular mechanisms by which it regulates homeostasis in mammals. Furthermore, a possible link between the disruption of the CCR4-NOT complex and various diseases will be discussed. Finally, we propose that the analysis of mice with each CCR4-NOT subunit knocked out is an effective strategy for clarifying its complicated functions and networks in mammals.

\section{INTRODUCTION}

Carbon catabolite repression 4 (CCR4)-negative on TATA-less (NOT) is a versatile complex that regulates various physiological processes in eukaryotes (Collart, 2003). Of its diverse functions, including transcriptional regulation and protein modification, deadenylation of mRNA has been particularly highlighted. Shortening of poly(A) tails leads to the degradation of mRNA via one of two ways, i.e., $5^{\prime}-3^{\prime}$ digestion by XRN1 after decapping by DCP1-DCP2 or $3^{\prime}-5^{\prime}$ digestion by the exosome, consequently suppressing gene expression (see Bartlam and Yamamoto, 2010; Doidge et al., 2012a for more details). In mammals, deadenylation is tightly regulated by various enzymes, such as poly(A)-specific ribonuclease (PARN), PAN2-PAN3, Nocturnin, and Angel1/2, as well as CCR4-NOT (Wiederhold and Passmore, 2010; Doidge etal., 2012a). Among these enzymes, PAN2-PAN3 and CCR4NOT are considered to be dominant in mammalian cells because cytoplasmic mRNA decay is reported to be conducted in two phases: an initial step by PAN2-PAN3 and a subsequent step by CCR4-NOT (Yamashita et al., 2005; Bartlam and Yamamoto, 2010).

CCR4 was originally identified as a gene required for the regulation of glucose-repressible alcohol dehydrogenase 2 (ADH2) in the yeast Saccharomyces cerevisiae (Denis, 1984). The Ccr4passociated factor (CAF1, also known as POP2) gene was found to be required for glucose derepression (Sakai et al., 1992). These two gene products were shown to form a large complex with Not proteins, which are related to the regulation of HIS3 transcription mainly through its TATA-less core promoter (Collart and Struhl, 1993, 1994). The core CCR4-NOT complex consists of Ccr4p, Caflp, five Not proteins (Notlp-Not5p), Caf40p, and Caf130p in yeast (Chen et al., 2001). Further study revealed that Ccr4p and Caflp are the major cytoplasmic mRNA deadenylases in yeast (Tucker et al., 2001; see below).

Initially, only the function of the CCR4-NOT complex in transcription was investigated because the core components of the complex were identified as possible transcriptional regulators (Collart, 2003). However, as mentioned above, mRNA silencing by the CCR4-NOT complex was then discovered. Thus, how the CCR4-NOT complex determines target mRNAs for degradation is now one of the more compelling questions. The CCR4-NOT complex interacts with various proteins, including the BTG/Tob family, RNA-binding proteins and miRNA-induced silencing complexes (miRISCs), to specify target mRNAs (Doidge et al., 2012a). Cytoplasmic polyadenylation element-binding protein (CPEB) binds specific mRNAs, to which Tob, as an adapter protein, mediates the recruitment of the CCR4-NOT deadenylase (Hosoda et al., 2011; Ogami et al., 2014). Other RNA-binding proteins, such as Nanos, Pumilio and fem-3 binding factor (PUF) proteins, Smaug, tristetrapolin (TTP), and Bicaudal-C (Bic-C), were reported to interact with the components of CCR4-NOT and cause the suppression of target mRNAs (Semotok et al., 2005; Goldstrohm et al., 2006, 2007; Chicoine et al., 2007; Suzuki et al., 2010, 2012; Sandler et al., 2011; Van Etten et al., 2012; Fabian et al., 2013; Joly et al., 2013; Bhandari et al., 2014). In addition, CCR4-NOT is involved in the microRNA (miRNA)-mediated deadenylation of mRNAs through interaction with the GW182 proteins (Behm-Ansmant et al., 2006; Braun et al., 2011; Chekulaeva et al., 2011; Fabian et al., 2011).

The CCR4-NOT components in yeast, except for Caf130p, are well conserved in mammals (Panepinto etal., 2013). CNOT1, 
CNOT2, CNOT4, and CNOT9 are mammalian orthologs of Not1p, Not2p, Not4p, and Caf40p, respectively. However, there are some compositional differences between the mammalian and yeast CCR4-NOT complexes. First, the E3 ubiquitin ligase CNOT4 does not stably associate with the mammalian CCR4-NOT complex (Lau et al., 2009). Second, there are four deadenylase subunits in the mammalian CCR4-NOT complex; yeast Ccr4p, which belongs to the exonuclease/endonuclease/phosphatase (EEP) family, is diverged into CNOT6 and CNOT6L, and Caflp, which belongs to the DEDD (Asp-Glu-Asp-Asp) family, is into CNOT7 and CNOT8. The expression of these paralogs was reported to be mutually exclusive in the human CCR4-NOT complex in HeLa S3 cells (Lau et al., 2009). Third, CNOT3 is a mammalian ortholog of two yeast Not proteins: Not3p and Not5p. Finally, orthologs of mammalian CNOT10 and C2orf29 (CNOT11) are missing in fungi (Mauxion et al., 2013; Panepinto et al., 2013). As the function of some CCR4-NOT components is different between yeast and mammals, it would be interesting to compare their biological roles across species. The structural organization and deadenylation machinery of the mammalian CCR4-NOT complex can be proposed as shown in Figures 1A,B, respectively.

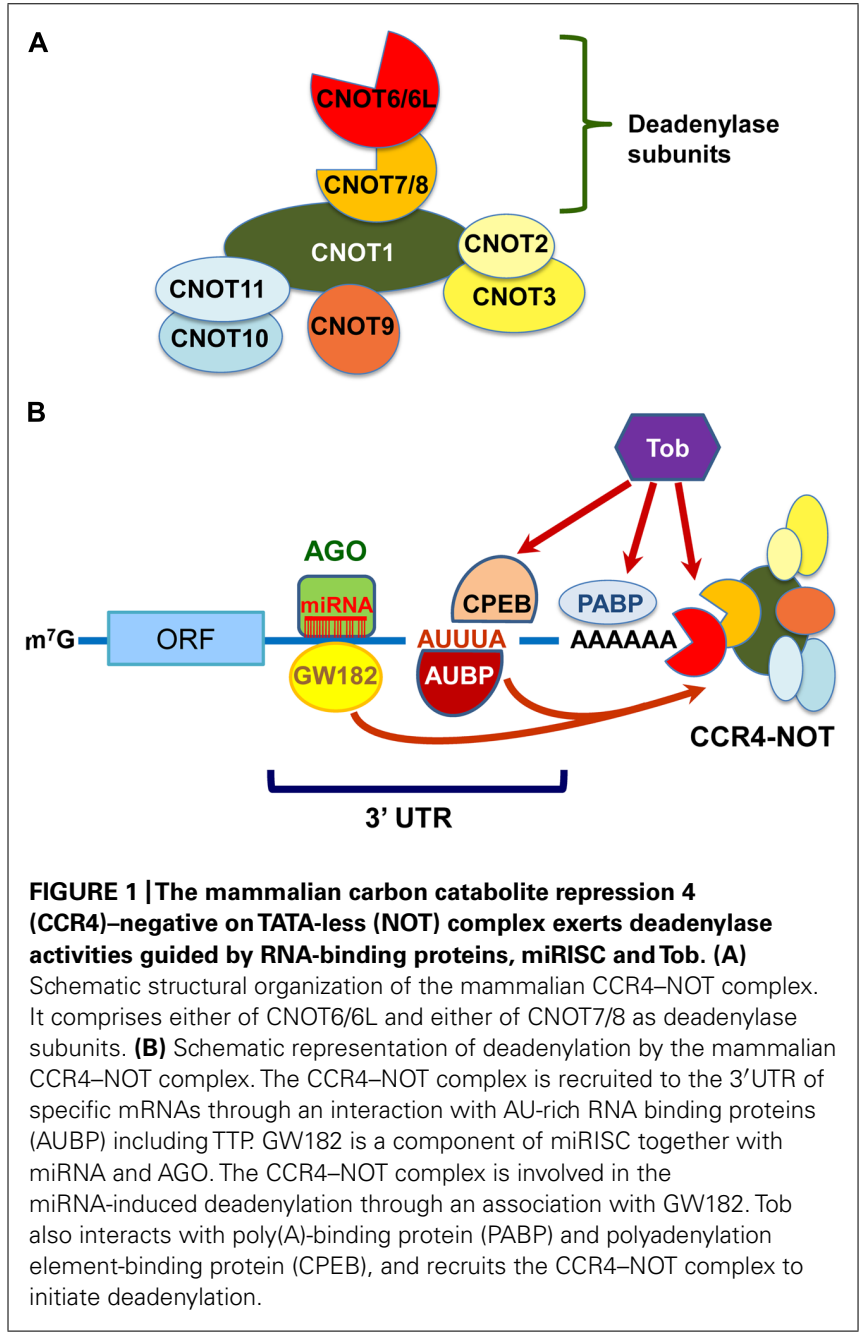

The CCR4-NOT complex is involved in various cellular mechanisms, including cell growth, DNA repair, mRNA export, histone methylation, and protein quality control in yeast (Collart, 2003; Mulder et al., 2005, 2007; Kerr etal., 2011; Halter et al., 2014). The CCR4-NOT complex is also implicated in the regulation of cell wall integrity, antifungal drug susceptibility, and developmental switches in pathogenic fungi (Panepinto et al., 2013). As we describe here, recent studies using cultured mammalian cells and genetically modified mice have revealed distinct roles of each subunit in wide-ranging physiological phenomena. Furthermore, deviation in the expression of CCR4-NOT has been found in various diseases based on analyses of human clinical specimens. In this review, we focus on the physiology of the mammalian CCR4-NOT complex and will occasionally refer to findings from non-mammalian systems.

\section{CNOT1}

CNOT1 is the largest subunit of the CCR4-NOT complex in molecular size and is a scaffold protein involved in the assembly of CCR4-NOT components (Figure 1A). From a structural point of view, CNOT1 can be divided into three main regions, namely the $\mathrm{N}$-terminal, the middle and the C-terminal, according to structural predictions and information (Bawankar et al., 2013). The $N$-terminal region of CNOT1 associates with CNOT11, which interacts with CNOT10 (Bawankar et al., 2013). The middle part of CNOT1, named the MIF4G (middle domain of eukaryotic initiation factor 4G) domain, directly interacts with CAF1a (CNOT7) or CAF1b (CNOT8; Basquin et al., 2012; Petit et al., 2012; Bawankar et al., 2013). In addition, CAF40 (CNOT9) interacts with the remaining residues of the middle portion of CNOT1, which comprises a DUF3819 domain (Bawankar et al., 2013; Chen et al., 2014; Mathys et al., 2014). Finally, the C-terminal region of CNOT1 associates with the CNOT2/CNOT3 complex (Bawankar et al., 2013; Boland et al., 2013). Through these interactions, CNOT1 provides a scaffold function for complex formation. Accordingly, depletion of CNOT1 destabilizes the complex, and each component is degraded, resulting in decreased levels of CNOT2, CNOT6L, CNOT7, and CNOT9, but not CNOT3, in HeLa cells (Ito et al., 2011a).

CNOT1 is an indispensable component for the deadenylase activities of the CCR4-NOT complex; however, CNOT1 does not possess any catalytic domains. Indeed, depletion of CNOT1 in HeLa cells compromises the deadenylase activities and decreases the number of P-bodies (Ito et al., 2011a), where mRNA decay is thought to take place (see review by Parker and Sheth, 2007). CNOT1-depleted cells undergo caspase-dependent apoptosis most likely through endoplasmic reticulum (ER) stress resulting from protein overproduction (Ito et al., 2011a).

CNOT1 also serves as a platform for the RNA-binding proteins that determine the specificity of the CCR4-NOT deadenylase for target mRNAs (Figure 1B). TTP, which is involved in AU-rich element (ARE)-mediated mRNA decay, directly interacts with CNOT1, and this interaction is required for the recruitment of CNOT7 to target mRNAs such as tumor necrosis factor- $\alpha$ (TNF- $\alpha$ ) to promote deadenylation (Sandler et al., 2011; Fabian et al., 2013). In addition, mammalian Nanos paralogs (Nanos1, Nanos2, and Nanos3) directly interact with the C-terminal domain of CNOT1 
(Suzuki et al., 2012; Bhandari et al., 2014). The interaction between Nanos2 and CNOT1 plays an important role in murine male germ cell development, most likely through regulation of the turnover of specific mRNAs, such as Stra8 (Suzuki et al., 2010, 2012). CNOT1 was also reported to interact with IGF2 mRNA-binding protein 1 (IGF2BP1, also known as IMP1; Hämmerle et al., 2013). In human liver cancer cells, CCR4-NOT takes part in the posttranscriptional repression of the long noncoding RNA highly $u$ p-regulated in liver cancer (HULC), to which IGF2BPs specifically bind (Hämmerle et al., 2013).

CNOT1 is a key player in linking the CCR4-NOT complex to miRNA-mediated repression (Figure 1B). Human GW182 proteins (also known as TNRC6A, TNRC6B, and TNRC6C), Argonaute (AGO)-binding partners and crucial effectors of miRISCs (Fabian and Sonenberg, 2012), were shown to directly interact with CNOT1 as well as PAN3 (Braun et al., 2011; Chekulaeva et al., 2011; Fabian et al., 2011). The GW182 proteins provide a platform for CCR4-NOT and PAN2-PAN3 to cause deadenylation of miRNA-target mRNAs (Braun et al., 2011; Chekulaeva et al., 2011; Fabian et al., 2011). In addition, CCR4-NOT is also required for silencing unadenylated RNA (Braun et al., 2011; Chekulaeva et al., 2011). Recent studies showed that the RNA helicase DDX6, a translational repressor and decapping activator, also interacts with CNOT1 through the MIF4G domain and this interaction is important for miRNA repression (Chen et al., 2014; Mathys et al., 2014). These findings propose that CCR4-NOT also plays an important role in miRNA-mediated translational suppression via recruitment of DDX6.

CNOT1 is commonly involved in mRNA decay in eukaryotes, including Drosophila melanogaster (Eulalio et al., 2007; Temme et al., 2010) and Schmidtea mediterranea (freshwater planarian) stem cells (Solana et al., 2013). Knockdown of planarian NOT1 increases stem cell specific-mRNAs with elongated poly(A) tails and leads to failure of cell differentiation (Solana et al., 2013). Remarkably, a single deletion of only the NOT1 gene among the CCR4-NOT components causes lethality in Saccharomyces cerevisiae, while double deletion of the CCR4 and CAF1 genes does not cause lethality (Maillet et al., 2000), which also suggests that CNOT1 provides not only a scaffold function for the CCR4-NOT deadenylase complex but also multiple other functions that are important for cellular homeostasis.

Transcriptional regulation is considered one of the most important functions of CNOT1, as Not1p is known to regulate transcription by RNA polymerase II in yeast (Lee et al., 1998). In MCF-7 human breast cancer cells that are positive for estrogen receptor $\alpha(\mathrm{ER} \alpha), \mathrm{CNOT} 1$ interacts with $\mathrm{ER} \alpha$ through the LXXLL motif and represses the ligand-dependent transcriptional activation of ER $\alpha$ (Winkler et al., 2006). CNOT1 can also interact with another member of the nuclear receptor superfamily, retinoid X receptor $\alpha(\mathrm{RXR} \alpha)$, and suppress RXR-mediated transcription in a ligand-dependent fashion (Winkler et al., 2006). In addition, CNOT1 as well as CNOT2 and CNOT3 suppresses the expression of trophectoderm transcription factors and plays a critical role in maintaining the state of human and mouse embryonic stem cells (ESCs), contrary to what occurs in planarian stem cells (Zheng et al., 2012; See details in Section "CNOT2 and CNOT3"). A genome-wide RNAi screen identified CNOT1 and CNOT2 as transcriptional and/or higherorder regulators of major histocompatibility complex class II molecules (MHC-II) in human melanoma MelJuSo cells (Paul et al., 2011).

Some clinical studies indicate that CNOT1 single nucleotide polymorphisms (SNPs) are relevant to physiological function, carcinogenesis, and the clinical outcome of disease. A locus in chromosome 16q21, which includes an intronic SNP within the CNOT1 gene, was newly identified as a modulator of the QT interval duration of the electrocardiogram in two independent studies (Newton-Cheh et al., 2009; Pfeufer et al., 2009). NOT1 was also identified as a key factor that was essential for heart functions using a genome-wide in vivo RNAi screen in Drosophila (Neely et al., 2010). In addition, SNPs within the CNOT1 and CNOT6 genes are associated with susceptibility to B-cell pediatric acute lymphoblastic leukemia (ALL; Gutierrez-Camino et al., 2014). The CNOT1 SNP in dendritic cells (DCs) of $18 \mathrm{HIV}$ patients was also associated with the response to DC-based immune therapy, as determined based on the plasma viral load (Moura et al., 2014). These findings suggest that CNOT1 is deeply involved in the functions of the cardiovascular, hematopoietic, and immune systems in humans.

Finally, Cnot1 knockout (KO) mice died during embryonic development (unpublished). Analyses of Cnot1 $\mathrm{KO}$ mice and heart or immunocyte-specific Cnot 1 conditional $\mathrm{KO}$ ( $\mathrm{cKO}$ ) mice would be an attractive strategy to clarify the molecular mechanism by which CNOT1 contributes to the maintenance of proper physiological functions in mammals. Findings from further studies are awaited.

\section{CNOT2 AND CNOT3}

CNOT2 and CNOT3 are structurally similar, having a unique conserved domain, i.e., the NOT-box, which is localized at the C-termini of CNOT2 and CNOT3 (Zwartjes et al., 2004). The NOT-box of CNOT2 is involved in transcriptional regulation through an interaction with histone deacetylase 3 (HDAC3), which is associated with the retinoic acid (RA) receptor, thyroidhormone receptor (SMRT), or nuclear hormone receptor corepressor (NCoR; Zwartjes et al., 2004; Jayne et al., 2006). The CNOT2 and CNOT3 proteins directly interact with each other in various eukaryotes (Ito et al., 2011b; Bawankar et al., 2013; Boland et al., 2013), and this interaction is achieved through their C-terminal regions comprising the NOT-boxes (Bawankar et al., 2013; Boland et al., 2013). In addition, CNOT2 mediates the interaction between CNOT3 and CNOT1 (Lau et al., 2009; Ito et al., 2011b; Boland etal., 2013). Consistently, depletion of CNOT2 in HeLa cells affects the integrity of the CCR4-NOT complex. Its depletion extensively decreases the expression level of CNOT3 and modestly decreases the expression of other CNOTs by destabilizing them (Ito et al., 2011b).

CNOT2 and CNOT3 do not possess catalytic domains, and there has been no report about their direct enzymatic activities. However, increasing evidence indicates that CNOT2 and CNOT3 function as positive modulators of the catalytic activities of the CCR4-NOT deadenylase and promote the degradation of target mRNAs. For instance, depletion of Not2p, Not3p, or Not5p suppresses cellular deadenylase activity, albeit slightly, and results in 
the stabilization of mRNAs in yeast (Tucker et al., 2002). It is noteworthy that this function of CNOT2 and CNOT3 is pertinent to various biological phenomena. A NOT2 or NOT5 mutation is synthetically lethal with a CAF1 or CCR4 deletion (Bai et al., 1999). By contrast, a NOT3 mutation with either a CAF1 or CCR4 deletion does not result in synthetic lethality (Bai et al., 1999), suggesting that Not5p is more essential than Not3p for the function of the CCR4-NOT complex in yeast.

Also, in higher eukaryotes, CNOT2 and CNOT3 regulate mRNA decay in various physiological processes. In Drosophila S2 cells, depletion of NOT2 affects the length of mRNA poly(A) tails (Temme et al., 2004). In addition, NOT3 directly interacts with the RNA-binding protein Bic- $\mathrm{C}$, which is required for maternal patterning in the Drosophila embryo, and thus contributes to recruitment of the catalytic subunit of the deadenylase to its target mRNAs (Chicoine et al., 2007). In mammals, CNOT3 is essential for embryonic viability because deficiency in CNOT3 results in lethality of mice at early embryonic stages (Neely et al., 2010; Morita et al., 2011). Moreover, Cnot $3^{+/-}$mice are lean and display resistance to high-fat-diet (HFD)-induced obesity compared to control mice due to enhanced metabolic rates and glucose tolerance (Morita et al., 2011). We clarified that a reduction in CNOT3 expression affects the stability of specific mRNAs encoding proteins mainly involved in energy metabolism, such as pyruvate dehydrogenase kinase, isozyme 4 (PDK4), and IGF binding protein 1 (IGFBP1), in the livers of $\mathrm{Cnot}^{+/-}$mice (Morita et al., 2011). Cnot $3^{+/-}$mice also show osteopenia and exacerbated aging-induced osteoporosis with enhanced bone resorption (Watanabe et al., 2014). It was also found that the mRNA expression of receptor activator of nuclear factor- $\kappa \mathrm{B}(R A N K)$, but not RANK ligand (RANKL), is up-regulated in $C n o t 3^{+/-}$mice and its mRNA stability is enhanced by Cnot3-deficiency in bone marrow-derived macrophages (Watanabe et al., 2014). These findings suggest that CNOT3-mediated regulation of the CCR4-NOT deadenylase is essential for a wide-range of physiological processes in mice.

In cultured mammalian cells, CNOT2 and CNOT3 reportedly affect cell cycle progression through the regulation of mRNA turnover. Knockdown of CNOT2 in HeLa cells impairs the deadenylase activity of the CCR4-NOT complex and decreases the number of P-bodies, as is the case for CNOT1 (Ito et al., 2011a,b). The cells, in turn, undergo caspase-dependent apoptosis, most likely due to the ER-stress induced by overproduction of the proteins that results from elevated levels of the stabilized mRNAs (Ito et al., 2011a,b). By contrast, knockdown of CNOT3 specifically increases the expression of MAD1 by stabilizing its mRNA and induces mitotic arrest of HeLa cells (Takahashi et al., 2012).

Transcriptional regulation is another important function of CNOT2 and CNOT3. Recently, a CNOT2-centered transcriptional network module was reported to be relevant for the susceptibility of breast cancer to metastasis (Faraji et al., 2014). CNOT2 also regulates the metastasis of murine breast cancer in a mouse xenograft model (Faraji et al., 2014). Regarding CNOT3, a genome-wide RNAi screen identified CNOT3 as one of the molecules that form a unique module in the transcriptional network and are essential for mouse ES cell self-renewal (Hu et al., 2009). CNOT2 and CNOT3 as well as CNOT1 contribute to the maintenance of mouse and human ESC identity, namely self-renewal and pluripotency, possibly through suppressing the expression of trophectoderm transcription factors (Zheng et al., 2012). Consistent with this, the expression of CNOT1, CNOT2, and CNOT3 closely correlates with the state of pluripotency (Zheng et al., 2012). It is noteworthy that single or even combined silencing of the deadenylase subunits does not affect the differentiation of ESCs, suggesting that transcriptional repression by CNOT1/CNOT2/CNOT3 is a key function in this phenomenon (Zheng et al., 2012). A global in vivo Drosophila RNAi screen identified NOT3 in addition to NOT1 as a critical regulator of heart function (Neely et al., 2010). In fact, Cnot $3^{+/-}$mice exhibit spontaneous impairment of cardiac contractility and develop severe cardiomyopathy in response to artificial cardiac stress by transverse aortic constriction (TAC) surgery (Neely et al., 2010). These phenomena are most likely due to decreased CNOT3 expression, resulting in the dysfunction of chromatin modification at the promoter regions of genes relevant to heart functions (Neely et al., 2010). However, in this case, it remains to be investigated whether CNOT3 also affects the stability of specific mRNAs in the heart (Neely et al., 2010).

Finally, several reports suggest that dysregulation of CNOT3 expression is relevant to a wide-range of human diseases. SNP analysis revealed that there is a significant association between CNOT3 polymorphism and cardiac repolarization duration (Neely et al., 2010) or susceptibility to ankylosing spondylitis (Díaz-Peña etal., 2012). In addition, CNOT3 was identified as a modifier of mutations in the causative gene of retinitis pigmentosa, PRPF31, which prevents the manifestation of this disease (Venturini et al., 2012). Importantly, CNOT3 was also identified as a tumor suppressor that is mutated in adult T-cell ALL, and its knockdown indeed caused tumors in a sensitized D. melanogaster model (De Keersmaecker et al., 2013). The mechanisms by which CNOT3 contributes to the prevention of carcinogenesis are unknown. As such, further studies are necessary to clarify the molecular functions of CNOT3, especially by addressing which of the two major mechanisms, mRNA decay or transcriptional regulation, mainly contributes to individual biological phenomena.

\section{CNOT6 AND CNOT6L}

The evolutionarily conserved protein CCR4 is one of the two deadenylase subunits of the yeast CCR4-NOT complex and belongs to the EEP superfamily (Collart and Panasenko, 2012; Winkler and Balacco, 2013). Yeast CCR4 (Ccr4p) was originally identified as a regulator of $\mathrm{ADH} 2$ in the yeast Saccharomyces cerevisiae (Denis, 1984). In a series of genetic studies, Ccr4p was shown to be involved in biological functions such as the DNA damage response (Collart and Panasenko, 2012).

CCR4 is characterized by the presence of two highly conserved domains: a $C$-terminal deadenylase domain and an aminoterminal leucine-rich repeat (LRR) domain. The LRR domain is essential for binding to CAF1 and incorporation into the CCR4NOT complex (Winkler and Balacco, 2013). However, there are some differences in the structure and composition of CCR 4 among species. First, the activation domain located in the $N$-terminalmost region of Saccharomyces cerevisiae Ccr4p is not present in 
its orthologs in other species of eukaryotes, including mammals (Morita et al., 2007). Second, there is only a single CCR4 in yeasts as well as in the metazoans D. melanogaster and Caenorhabditis elegans, while two orthologs, CNOT6 (also known as CCR4a) and CNOT6L (also known as CCR4b), exist in vertebrates, including Xenopus laevis, Danio rerio, Mus musculus, and Homo sapiens (Dupressoir et al., 2001; Morita et al., 2007; Cooke et al., 2010; Winkler and Balacco, 2013).

CCR4 has been implicated in a variety of biological functions, as inferred from genetic and RNA interference experiments in many biological species (Goldstrohm and Wickens, 2008). It is worth noting that CCR4 family proteins are not essential for the viability of the organisms studied to date (Goldstrohm and Wickens, 2008). Among the various physiological roles of CCR4 over multiple species, the role of CCR4 during the early development of various organisms has been well characterized. For instance, mutations in the Drosophila gene twin, which encodes CCR4, affect female reproductive capacity and display defects in the self-renewal of germline stem cells (Temme et al., 2004; Morris et al., 2005; Zaessinger et al., 2006; Joly et al., 2013). In particular, the expression of the mei-P26 mRNA is specifically regulated by CCR4 through the RNA-binding proteins Nanos and Pumilio during germline stem cell self-renewal in Drosophila (Joly et al., 2013). By contrast, the poly(A) tail length of mRNAs was globally increased by depletion of CCR4 in C. elegans germ cells, suggesting the CCR4-NOT complex plays a key role in general mRNA metabolism during early development in C. elegans (Nousch et al., 2013).

The mammalian CCR4 orthologs CNOT6 and CNOT6L have also been recently studied. The crystal structural analysis of human CNOT6L revealed that its deadenylase domain is remarkably similar to that of other EEP proteins and selectively interacts with poly(A) residues (Wang et al., 2010). In addition, in vitro deadenylation assays revealed that CNOT6L prefers poly(A) RNA to poly(A) DNA, indicating that CNOT6L preferentially functions as deadenylase of mRNAs (Wang et al., 2010).

CNOT6 and CNOT6L were reported to be mutually exclusive in the human CCR4-NOT complex in HeLa S3 cells, implying that the two paralogs have compensatory roles in mRNA deadenylation (Lau et al., 2009). Indeed, both CNOT6 and CNOT6L contribute to efficient cell growth of MCF-7 cells (Mittal et al., 2011). It should be noted that microarray analysis demonstrated that depletion of CNOT6 has little effect on mRNA levels compared to depletion of CNOT6L (Mittal et al., 2011). In addition, the tissue distribution of CNOT6 and CNOT6L in mice is distinctive. CNOT6L is ubiquitously expressed in various tissues, while CNOT6 is highly expressed in testis, ovary, thymus, and spleen (Chen et al., 2011). Moreover, CNOT6L, but not CNOT6, influences cell proliferation of murine fibroblasts (NIH3T3 cells) through regulation of the $C d k n 1 b$ mRNA (Morita et al., 2007). These findings indicate that CNOT6 and CNOT6L have discrete roles in addition to a mutually overlapping role in cells. Both Cnot6 KO and Cnot6l KO mice are viable and fertile (unpublished). Further studies with these $\mathrm{KO}$ mice would unravel the physiological differences between CNOT6 and CNOT6L.

Recent study demonstrated that significant copy number loss of CNOT6L was present in human colon adenocarcinoma samples
(Tay et al., 2011). The expression of CNOT6L and CNOT7 was reportedly down-regulated in samples of leukemia cells taken from ALL and AML (acute myeloid leukemia) patients compared to normal blood cells, while the expression of CNOT6 was slightly up-regulated (Maragozidis et al., 2012). In addition, SNPs in the CNOT1 and CNOT6 genes were significantly associated with B-cell ALL susceptibility (Gutierrez-Camino et al., 2014). However, the involvement of CNOT6 and CNOT6L in the onset and progression of cancer is still not clarified. Apparently, further studies investigating the function of CNOT6 and CNOT6L in various diseases are required.

Finally, there are additional members of the mammalian CCR4 family: Nocturnin (CCR4c), Angel1 (CCR4d), Angel2 (CCR4e), and 2'phosphodiesterase (2'PDE; Goldstrohm and Wickens, 2008). These proteins lack a LRR domain, which indicates that they are not always components of the CCR4-NOT complex (Godwin et al., 2013). Their physiological functions are still largely unknown, and we would like to omit these details in this review (see Poulsen et al., 2011; Godwin et al., 2013).

\section{CNOT7 AND CNOT8}

Caf1, which possesses a ribonuclease (RNase) D domain from the DEDD superfamily, is the other core deadenylase subunit of the CCR4-NOT complex (Collart and Panasenko, 2012; Winkler and Balacco, 2013). Initially, yeast Caf1 (Caf1p) was identified as a possible transcriptional regulator required for glucose-derepression in Saccharomyces cerevisiae (Sakai et al., 1992). Subsequently, Tucker et al. found that Caflp together with Ccr4p is a major cytoplasmic mRNA deadenylase (Tucker et al., 2001). Since then, deadenylation by Caf1 has been intensively studied.

The amino acid sequences of CAF1 orthologs are highly conserved across species (Winkler and Balacco, 2013). In Trypanosoma brucei, no ortholog for CCR4 was found, and CAF1 is the only deadenylase unit of the trypanosome CCR4-NOT complex, which is crucial for the deadenylation and subsequent degradation of many mRNAs (Schwede et al., 2008). In Drosophila, although both CCR4 and CAF1 are involved in the regulation of bulk mRNA poly(A) deadenylation, the catalytic activity of Caf1 is considered more potent than that of CCR4 (Temme et al., 2004, 2010). By contrast, Ccr4p is the dominant deadenylase, and Caflp is dispensable in the presence of the catalytic activity of Ccr4p in yeast (Tucker et al., 2002). However, there remains a possibility that Caflp is responsible for the regulation of deadenylation of specific mRNAs in yeast (Tucker et al., 2002; Wiederhold and Passmore, 2010). Thus, the biological significance of CAF1 differs among eukaryotes, even though the molecular structures of its orthologs are well conserved.

In mammals including humans, there are two orthologs of CAF1: CNOT7 (hCAF1a) and CNOT8 (hCAF1b). Amino acid sequence analysis of human CNOT7 and CNOT8 demonstrated that there is a high degree of homology between them (Bianchin et al., 2005; Winkler and Balacco, 2013). Human CNOT7 and CNOT8 bind the middle region of CNOT1 through its MIF4G domain, which is composed of antiparallel pairs of $\alpha$-helices (Basquin et al., 2012; Petit et al., 2012; Bawankar et al., 2013). In addition, yeast two-hybrid analysis revealed that CNOT6 directly binds CNOT7 and CNOT8, but not CNOT1 (Lau et al., 2009). 
These findings suggest that the interaction of CNOT7 and CNOT8 with CNOT1 is essential for CNOT6 and CNOT6L to be integrated into the CCR4-NOT complex and exert their deadenylation functions.

Both CNOT7 and CNOT8 are ubiquitously expressed in adult mouse tissues (Chen et al., 2011). It is worth mentioning that high expression levels of both CNOT7 and CNOT8 proteins were detected, especially in thymus, spleen, ovary, and testis, and their expression patterns were similar (Chen et al., 2011). The combined depletion of CNOT7 and CNOT8 as well as CNOT6 and CNOT6L resulted in the strong inhibition of ARE-containing $\beta$-globin mRNA decay in human HTGM5 cells (Schwede et al., 2008). In addition, CNOT7 and CNOT8 were reported to be mutually exclusive in the CCR4-NOT complex in HeLa S3 cells, as is the case for CNOT6 and CNOT6L (Lau et al., 2009). These findings suggest that the Cafl paralogs are important for mRNA deadenylation in mammals, and each has overlapping functions. Indeed, CNOT7 and CNOT8 are required for the proliferation of MCF7 human breast cancer cells and redundantly repress the antiproliferative gene PMP22 through their deadenylase activities (Aslam et al., 2009).

It is noteworthy that CNOT7 and CNOT8 are capable of binding BTG/Tob proteins (BTG1, BTG2/PC3/Tis21, BTG3/ANA, BTG4/PC3B, Tob1, and Tob2), which are characterized by the presence of a conserved BTG domain and regulate cell cycle progression (Winkler, 2010; Winkler and Balacco, 2013). The interaction with CNOT7/CNOT8 is required for the anti-proliferative activities of Tob proteins in various types of mammalian cells (Horiuchi et al., 2009; Doidge et al., 2012b; Ezzeddine et al., 2012). Importantly, Tob functions as a guide molecule for the CCR4-NOT complex to target mRNAs through an interaction with CNOT7 (Doidge etal., 2012a). Tob is capable of associating with the general RNA-binding protein, poly(A)-binding protein (PABP), and recruits the CCR4-NOT complex to initiate mRNA decay (Funakoshi et al., 2007). Moreover, Tob also mediates the recruitment of the CCR4-NOT deadenylase to specific mRNAs, such as MYC, to which CPEB proteins bind (Hosoda et al., 2011; Ogami etal., 2014). There are some reports that Tob proteins and BTG2 activate the deadenylation of mRNAs in cultured cells (Ezzeddine et al., 2007, 2012; Mauxion et al., 2008). However, there is an opposing report that BTG2 inhibits deadenylation by CNOT7/CNOT8 in vitro (Yang et al., 2008). As such, regulation of the deadenylation activity of CNOT7/CNOT8 by BTG/Tob proteins might be dependent on extracellular and intracellular circumstances. Further studies should clarify this complicated molecular network.

CNOT7 is known to be deeply involved in cellular signaling pathways through interacting with various types of proteins in addition to BTG/Tob proteins. CNOT7 regulates class I and II interferon (IFN) pathways by controlling signal transducer and activator of transcription 1 (STAT1) trafficking through an interaction with its latent form and degrading STAT1-regulated mRNAs through its deadenylase activity (Chapat et al., 2013). Remarkably, Cnot7-deficiency results in physiological anomalies in mice. Cnot7 KO male mice exhibit severe infertility associated with oligo-astheno-teratozoospermia (Berthet et al., 2004; Nakamura et al., 2004). CNOT7 physically interacts with RXR beta (Rxrb) and is involved in spermatogenesis by functioning as a coregulator of Rxrb in testicular somatic cells (Nakamura et al., 2004). Moreover, Cnot7 KO mice show increased bone mass, and CNOT7 was found to function as a suppressor of bone morphogenetic protein (BMP) signaling (Washio-Oikawa et al., 2007). These findings suggest that CNOT7 regulates the balance in various biological processes in mammals. Both Cnot7 and Cnot8 $\mathrm{KO}$ mice would be useful tools to clarify the functional difference between CNOT7 and CNOT8.

There are some reports that CNOT7 and CNOT8 are relevant to the development and progression of cancer. The expression of CNOT7 was down-regulated in ALL and AML patients (Maragozidis etal., 2012), and the expression of CNOT8 was elevated in primary colorectal carcinoma and metastatic legions compared to the normal mucosa (Seiden-Long et al., 2006). It remains to be investigated how CNOT7 and CNOT8 contribute to the development and progression of cancer.

\section{CNOT9}

Rcd1 (required for cell differentiation 1) was originally identified as a factor essential for nitrogen starvation-induced sexual differentiation in the fission yeast Schizosaccharomyces pombe (Okazaki et al., 1998). Shortly after this discovery, its Schizosaccharomyces cerevisiae homolog, Caf40, was identified by mass spectrometry analysis as a component of the CCR4-NOT complex (Chen et al., 2001). The amino acid sequences of Rcd1/Caf40 orthologs are highly conserved among eukaryotes (Okazaki et al., 1998; Garces et al., 2007).

CNOT9, the mammalian ortholog of Rcd1 and Caf40 (Collart and Timmers, 2004), acts as a transcriptional cofactor that plays a critical role in RA-induced differentiation of F9 embryonic teratocarcinoma cells and in lung development, through an association with a transcription complex containing activation transcription factor-2 (ATF-2) and RA receptors (RARs) in an RA-dependent fashion (Hiroi et al., 2002). CNOT9 interacts with nuclear hormone receptor transcriptional coactivator (NRC)interacting factor 1 (NIF-1; Garapaty et al., 2008). NIF-1 interacts with NRC, which is a coactivator of nuclear hormone receptors, and regulates its activities (Mahajan etal., 2002). CNOT9 also interacts with c-Myb, which is a proto-oncogene product and transcription factor involved in myeloid cell differentiation (Haas et al., 2004). In addition, CNOT9 was isolated as an erythropoietinresponsive gene that is potentially important for hematopoietic cell development (Gregory et al., 2000). Thus, the involvement of CNOT9 in the control of growth and differentiation of mammalian tissues, especially of the hematopoietic system, may be highlighted.

Although CNOT9 has not been shown to carry deadenylase activity, its involvement in deadenylation and/or mRNA decay was recently implicated by structural analysis (Chen et al., 2014; Mathys et al., 2014). CNOT9 comprises six armadillo repeats (ARM repeat domain) and forms a homodimer or alternatively interacts with CNOT1 (Garces etal., 2007; Chen etal., 2014; Mathys etal., 2014). The interaction takes place between the ARM repeat domain of CNOT9 and the DUF3819 domain of CNOT1, and the consequent binary complex provides binding sites for GW182 (Chen et al., 2014; Mathys et al., 2014). The ARM 
repeat domain is known to be involved in protein-protein interactions, as described above. Interestingly, a recent study revealed that the ARM repeat domain of $\beta$-catenin is associated with RNA binding and forming RNA-protein complexes (Kim et al., 2012), which suggests that CNOT9 is also capable of interacting with RNA through the ARM repeat domain. Consistently, a previous in vitro binding assay demonstrated the interaction between CNOT9 and single-stranded DNA polymers, i.e., $\operatorname{poly}(\mathrm{dC})$, poly $(\mathrm{dG})$, and poly(dT), but not poly(dA) (Garces et al., 2007).

Acting as a transcriptional regulator and/or an mRNA silencing protein, CNOT9 is expected to play important roles in various biological events in mammals. Increasing data indicate that the aberrant expression of CNOT9 is relevant to disease, as described below. First, some evidence suggests that CNOT9 is involved in mammary carcinogenesis. CNOT9 is frequently up-regulated in human breast cancer specimens and cell lines (Ajiro et al., 2009), and its expression is extremely low in normal human tissues, except testis, which suggests that CNOT9 is a cancer antigen (Ajiro et al., 2009). It should be noted, however, that the expression of CNOT9 is readily detectable in various murine adult tissues, including testis, ovary, thymus, brain, and lung (Chen et al., 2011). Short hairpin RNA (shRNA)-mediated suppression of CNOT9 drastically suppresses the proliferation of breast cancer cells (Ajiro et al., 2009). In addition, CNOT9 forms a large complex consisting of Grb10, Grb10-interacting protein 1 (GIGYF1), GIGYF2, and Akt, all of which are components of epidermal growth factor receptor (EGFR) downstream signaling (Ajiro et al., 2010). The Akt phosphorylation that is induced by EGFR signaling is attenuated in the absence of CNOT9 (Ajiro etal., 2010), which suggests that CNOT9 enhances EGFR signaling and contributes to carcinogenesis and progression of cancer, particularly in mammary glands. Second, Cnot9-deficient mice are embryonic lethal (unpublished). Further analyses of Cnot 9 null and cKO mice would provide insights into how CNOT9 regulates physiological processes through interactions with various types of molecules.

\section{CNOT10 AND CNOT11}

CNOT10 is a unique component of the CCR4-NOT complex in that its orthologs exist in eukaryotes, except in fungi (Bartlam and Yamamoto, 2010; Panepinto et al., 2013). Recent proteomic analysis uncovered that C2orf29 stably associates with the human CCR4-NOT complex (Lau et al., 2009). Ortholog of C2orf29 is also found in all eukaryotes examined except in yeast
(Mauxion et al., 2013). In the vertebrate CCR4-NOT complex, C2orf29 together with CNOT10 form a module that interacts with CNOT1. This interaction takes place through direct binding of C2orf29 with the $N$-terminus of CNOT1 in D. melanogaster and human cells (Bawankar et al., 2013; Mauxion et al., 2013). In addition, expression of CNOT10 and C2orf29 reciprocally stabilizes each other. C2orf29 was proposed to be renamed CNOT11 and is now recognized as a bona fide subunit of the CCR4-NOT complex.

Regarding the function of CNOT10/CNOT11, its ability to promote deadenylation of bound mRNAs was shown in tethering assays to be most likely due to recruitment of the catalytic module (Bawankar et al., 2013). However, depletion of CNOT11 did not affect the deadenylation rate of a $\beta$-globin mRNA reporter in human cells (Mauxion et al., 2013), suggesting that CNOT11 itself is not directly involved in deadenylation. In contrast, depletion of CNOT10 impaired the deadenylation of various mRNAs and inhibited the proliferation of trypanosomes (Färber et al., 2013). This discrepancy in the role of CNOT10/CNOT11 might result from constitutional and structural differences between the human and trypanosome CCR4-NOT complexes. Indeed, depletion of CNOT10 decreased the level of CNOT1 in trypanosomes, while this phenomenon was not observed in human cells (Färber et al., 2013). This finding suggests that the role of the CNOT10/CNOT11 module could differ among species, even though the module is conserved. Finally, Cnot10-deficiency in mice causes embryonic lethality (unpublished). The generation and analyses of Cnot11 $\mathrm{KO}$ mice would be necessary in future studies.

\section{CONCLUDING REMARKS AND PERSPECTIVES}

The core members of the CCR4-NOT complex were originally identified and characterized in yeast (Collart, 2003). Recent studies, including structural and biochemical analyses, have unveiled that the mammalian CCR4-NOT complex harbors both conserved and mammalian-specific functions. In addition, each subunit has distinct multifunctional roles through interactions with a wide-range of molecules. By contrast, the deadenylase units, namely CNOT6/CNOT6L and CNOT7/CNOT8, have overlapping functions. The biological significance of the versatile and yet conserved roles, to a certain extent, has not been well appreciated, although this complexity makes research on the CCR4-NOT complex intriguing. Many questions remain to be addressed. For instance, what are the roles of CNOT10 and CNOT11 in the murine and human CCR4-NOT complexes?

Table 1 | Phenotypes of KO mice of the CCR4-NOT complex subunits.

\begin{tabular}{lllll}
\hline Gene & Genotype & Dysregulation in the molecular functions & Biological anomaly & Reference \\
\hline Cnot3 & $-1-$ & Unknown & Embryonic lethality (E6.5) & Neely et al. (2010), Morita et al. (2011) \\
& $+1-$ & Up-regulation of Pdk4 and Igfbp1 mRNA & Leanness & Morita etal. (2011) \\
& & Up-regulation of Rank mRNA & Osteopenia & Watanabe et al. (2014) \\
& & Transcriptionally inactive chromatin & Cardiac dysfunction & Neely et al. (2010) \\
Cnot7 & $-1-$ & Impaired Rxrb signaling & Male sterility & Berthet et al. (2004), Nakamura et al. (2004) \\
& & Enhanced BMP signaling & Increased bone mass & Washio-Oikawa et al. (2007)
\end{tabular}


Strikingly, deficiency in each component of the CCR4-NOT complex, including CNOT1, CNOT3, CNOT9, and CNOT10, but not the deadenylase subunits CNOT6, CNOT6L, and CNOT7, in mice leads to embryonic lethality, suggesting that the CCR4-NOT complex is indispensable for embryonic development (unpublished). We are currently investigating whether Cnot2 $\mathrm{KO}$ and Cnot8 KO mice also exhibit developmental anomalies. Furthermore, the organ-specific functions of each CCR4-NOT component in mammals remain to be elucidated and could be addressed by generating $\mathrm{CKO}$ mice of each subunit in various organs. All the phenotypes of KO mice of each CCR4-NOT subunit reported are summarized in Table 1. Further analyses of null and cKO mice will help to clarify the inscrutable functions of the CCR4-NOT complex.

In summary, the CCR4-NOT complex globally regulates mRNA metabolism from transcriptional initiation to mRNA degradation. Thus, the CCR4-NOT complex has great influence on biological events via the regulation of consequent protein expression. It is becoming clear that there is a relationship between the CCR4-NOT complex and various diseases. Dissection of the physiological processes that are regulated by CCR4-NOT, a mRNA metabolism-controlling machinery, would be a novel and intriguing approach to elucidate the cause of disease, both at onset and upon progression. Further progress in the research on mRNA metabolism regulated by CCR4-NOT is anticipated.

\section{ACKNOWLEDGMENTS}

We would like to thank all of the members of Cell Signal Unit at the Okinawa Institute of Science and Technology Graduate University. This research was supported by KAKENHI (Grants-inAid for Scientific Research; 21229006 and for Scientific Research on Innovative Areas; 25121734 ) from the Japan Society for the Promotion of Science. This research was also supported by KAKENHI (Grants-in-Aid for Research Activity Start-up 24890289 to Yo-Taro Shirai; and for Young Scientists 90637589 to Akinori Takahashi) and Canadian Diabetes Association Postdoctoral Fellowship (to Masahiro Morita).

\section{REFERENCES}

Ajiro, M., Katagiri, T., Ueda, K., Nakagawa, H., Fukukawa, C., Lin, M. L., et al. (2009). Involvement of RQCD1 overexpression, a novel cancer-testis antigen in the Akt pathway in breast cancer cells. Int. J. Oncol. 35, 673-681. doi: 10.3892/ijo_00000379

Ajiro, M., Nishidate, T., Katagiri, T., and Nakamura, Y. (2010). Critical involvement of RQCD1 in the EGFR-Akt pathway in mammary carcinogenesis. Int. J. Oncol. 37, 1085-1093. doi: 10.3892/ijo_00000760

Aslam, A., Mittal, S., Koch, F., Andrau, J. C., and Winkler, G. S. (2009). The CCR4-NOT deadenylase subunits CNOT7 and CNOT8 have overlapping roles and modulate cell proliferation. Mol. Biol. Cell 20, 3840-3850. doi: 10.1091/mbc.E09-02-0146

Bai, Y., Salvadore, C., Chiang, Y. C., Collart, M. A., Liu, H. Y., and Denis, C. L. (1999). The CCR4 and CAF1 proteins of the CCR4-NOT complex are physically and functionally separated from NOT2, NOT4, and NOT5. Mol. Cell. Biol. 19, 6642-6651.

Bartlam, M., and Yamamoto, M. (2010). The structural basis for deadenylation by the CCR4-NOT complex. Protein Cell 25, 443-452. doi: 10.1007/s13238-0100060-8

Basquin, J., Roudko, V. V., Rode, M., Basquin, C., Séraphin, B., and Conti, E. (2012). Architecture of the nuclease module of the yeast Ccr4-not complex: the Not1-Caf1-Ccr4 interaction. Mol. Cell 48, 207-218. doi: 10.1016/j.molcel.2012. 08.014
Bawankar, P., Loh, B., Wohlbold, L., Schmidt, S., and Izaurralde, E. (2013). NOT10 and C2orf29/NOT11 form a conserved module of the CCR4-NOT complex that docks onto the NOT1 N-terminal domain. RNA Biol. 10, 228-244. doi: 10.4161/rna.23018

Behm-Ansmant, I., Rehwinkel, J., Doerks, T., Stark, A., Bork, P., and Izaurralde, E. (2006). mRNA degradation by miRNAs and GW182 requires both CCR4:NOT deadenylase and DCP1:DCP2 decapping complexes. Genes Dev. 20, 1885-1898. doi: $10.1101 / \operatorname{gad} .1424106$

Berthet, C., Morera, A. M., Asensio, M. J., Chauvin, M. A., Morel, A. P., Dijoud, F., et al. (2004). CCR4-associated factor CAF1 is an essential factor for spermatogenesis. Mol. Cell. Biol. 24, 5808-5820. doi: 10.1128/MCB.24.13.5808-58 20.2004

Bhandari, D., Raisch, T., Weichenrieder, O., Jonas, S., and Izaurralde, E. (2014). Structural basis for the Nanos-mediated recruitment of the CCR4NOT complex and translational repression. Genes Dev. 28, 888-901. doi: 10.1101/gad.237289.113

Bianchin, C., Mauxion, F., Sentis, S., Séraphin, B., and Corbo, L. (2005). Conservation of the deadenylase activity of proteins of the Caf1 family in human. RNA 11, 487-494. doi: 10.1261/rna.7135305

Boland, A., Chen, Y., Raisch, T., Jonas, S., Kuzuoğlu-Öztürk, D., and Wohlbold, L. et al. (2013). Structure and assembly of the NOT module of the human CCR4NOT complex. Nat. Struct. Mol. Biol. 20, 1289-1297. doi: 10.1038/nsmb.2681

Braun, J. E., Huntzinger, E., Fauser, M., and Izaurralde, E. (2011). GW182 proteins directly recruit cytoplasmic deadenylase complexes to miRNA targets. Mol. Cell 44, 120-133. doi: 10.1016/j.molcel.2011.09.007

Chapat, C., Kolytcheff, C., Le Romancer, M., Auboeuf, D., De La Grange, P., Chettab, K., et al. (2013). hCAF1/CNOT7 regulates interferon signaling by targeting STAT1. EMBO J. 32, 688-700. doi: 10.1038/emboj.2013.11

Chekulaeva, M., Mathys, H., Zipprich, J. T., Attig, J., Colic, M., Parker, R., et al. (2011). miRNA repression involves GW182-mediated recruitment of CCR4-NOT through conserved W-containing motifs. Nat. Struct. Mol. Biol. 18, 1218-1226. doi: $10.1038 / \mathrm{nsmb} .2166$

Chen, C., Ito, K., Takahashi, A., Wang, G., Suzuki, T., Nakazawa, T., et al. (2011). Distinct expression patterns of the subunits of the CCR4-NOT deadenylase complex during neural development. Biochem. Biophys. Res. Commun. 411, 360-364. doi: 10.1016/j.bbrc.2011.06.148

Chen, J., Rappsilber, J., Chiang, Y. C., Russell, P., Mann, M., and Denis, C. L. (2001). Purification and characterization of the 1.0 MDa CCR4-NOT complex identifies two novel components of the complex. J. Mol. Biol. 314, 683-694. doi: 10.1006/jmbi.2001.5162

Chen, Y., Boland, A., Kuzuoğlu-Öztürk, D., Bawankar, P., Loh, B., Chang, C. T., et al. (2014). A DDX6-CNOT1 complex and W-binding pockets in CNOT9 reveal direct links between miRNA target recognition and silencing. Mol. Cell 54, 737750. doi: 10.1016/j.molcel.2014.03.034

Chicoine, J., Benoit, P., Gamberi, C., Paliouras, M., Simonelig, M., and Lasko, P. (2007). Bicaudal-C recruits CCR4-NOT deadenylase to target mRNAs and regulates oogenesis, cytoskeletal organization, and its own expression. Dev. Cell 13, 691-704 doi: 10.1016/j.devcel.2007.10.002

Collart, M. A. (2003). Global control of gene expression in yeast by the Ccr4-Not complex. Gene 313, 1-16. doi: 10.1016/S0378-1119(03)00672-3

Collart, M. A., and Panasenko, O. O. (2012). The Ccr4-Not complex. Gene 492, 42-53. doi: 10.1016/j.gene.2011.09.033

Collart, M. A., and Struhl, K. (1993). CDC39, an essential nuclear protein that negatively regulates transcription and differentially affects the constitutive and inducible HIS3 promoters. EMBO J. 12, 177-186.

Collart, M. A., and Struhl, K. (1994). NOT1(CDC39), NOT2(CDC36), NOT3, and NOT4 encode a global-negative regulator of transcription that differentially affects TATA-element utilization. Genes Dev. 8, 525-537. doi: 10.1101/gad.8.5.525 Collart, M. A., and Timmers, H. T. (2004). The eukaryotic Ccr4-not complex: a regulatory platform integrating mRNA metabolism with cellular signaling pathways? Prog. Nucleic Acid Res. Mol. Biol. 77, 289-322. doi: 10.1016/S00796603(04)77008-7

Cooke, A., Prigge, A., and Wickens, M. (2010). Translational repression by deadenylases. J. Biol. Chem. 285, 28506-28513. doi: 10.1074/jbc.M110.150763

De Keersmaecker, K., Atak, Z. K., Li, N., Vicente, C., Patchett, S., Girardi, T., et al. (2013). Exome sequencing identifies mutation in CNOT3 and ribosomal genes RPL5 and RPL10 in T-cell acute lymphoblastic leukemia. Nat. Genet. 45, 186-190. doi: 10.1038/ng.2508 
Denis, C. L. (1984). Identification of new genes involved in the regulation of yeast alcohol dehydrogenase II. Genetics 108, 833-844.

Díaz-Peña, R., Aransay, A. M., Suárez-Álvarez, B., Bruges-Armas, J., RodríguezEzpeleta, N., Regueiro, M., et al. (2012). A high density SNP genotyping approach within the $19 \mathrm{q} 13$ chromosome region identifies an association of a CNOT3 polymorphism with ankylosing spondylitis. Ann. Rheum. Dis. 71, 714-717. doi 10.1136/annrheumdis-2011-20066

Doidge, R., Mittal, S., Aslam, A., and Winkler, G. S. (2012a). Deadenylation of cytoplasmic mRNA by the mammalian Ccr4-Not complex. Biochem. Soc. Trans. 40, 896-901. doi: 10.1042/BST20120074

Doidge, R., Mittal, S., Aslam, A., and Winkler, G. S. (2012b). The anti-proliferative activity of BTG/TOB proteins is mediated via the Cafla (CNOT7) and Caflb (CNOT8) deadenylase subunits of the Ccr4-not complex. PLoS ONE 7:e51331. doi: 10.1371/journal.pone.0051331

Dupressoir, A., Morel, A. P., Barbot, W., Loireau, M. P., Corbo, L., and Heidmann, T. (2001). Identification of four families of yCCR4- and $\mathrm{Mg}^{2+}$ dependent endonuclease-related proteins in higher eukaryotes, and characterization of orthologs of yCCR4 with a conserved leucine-rich repeat essential for hCAF1/hPOP2 binding. BMC Genomics 2:9. doi: 10.1186/1471-21 64-2-9

Eulalio, A., Behm-Ansmant, I., Schweizer, D., and Izaurralde, E. (2007). P-body formation is a consequence, not the cause, of RNA-mediated gene silencing. Mol. Cell. Biol. 27, 3970-3981. doi: 10.1128/MCB.00128-07

Ezzeddine, N., Chang, T. C., Zhu, W., Yamashita, A., Chen, C. Y., Zhong, Z., et al. (2007). Human TOB, an antiproliferative transcription factor, is a poly(A)-binding protein-dependent positive regulator of cytoplasmic mRNA deadenylation. Mol. Cell. Biol. 27, 7791-7801. doi: 10.1128/MCB.012 54-07

Ezzeddine, N., Chen, C. Y., and Shyu, A. B. (2012). Evidence providing new insights into TOB-promoted deadenylation and supporting a link between TOB's deadenylation-enhancing and antiproliferative activities. Mol. Cell. Biol. 32, 1089-1098. doi: 10.1128/MCB.06370-11

Fabian, M. R., Cieplak, M. K., Frank, F., Morita, M., Green, J., Srikumar, T., et al. (2011). miRNA-mediated deadenylation is orchestrated by GW182 through two conserved motifs that interact with CCR4-NOT. Nat. Struct. Mol. Biol. 18, 1211 1217. doi: $10.1038 / \mathrm{nsmb} .2149$

Fabian, M. R., Frank, F., Rouya, C., Siddiqui, N., Lai, W. S., Karetnikov, A., et al (2013). Structural basis for the recruitment of the human CCR4-NOT deadenylase complex by tristetraprolin. Nat. Struct. Mol. Biol. 20, 735-739. doi: $10.1038 / \mathrm{nsmb} .2572$

Fabian, M. R., and Sonenberg, N. (2012). The mechanics of miRNA-mediated gene silencing: a look under the hood of miRISC. Nat. Struct. Mol. Biol. 19, 586-593. doi: $10.1038 / \mathrm{nsmb} .2296$

Faraji, F., Hu, Y., Wu, G., Goldberger, N. E., Walker, R. C., Zhang, J., et al. (2014). An integrated systems genetics screen reveals the transcriptional structure of inherited predisposition to metastatic disease. Genome Res. 24, 227-240. doi $10.1101 /$ gr.166223.113

Färber, V., Erben, E., Sharma, S., Stoecklin, G., and Clayton, C. (2013). Trypanosome CNOT10 is essential for the integrity of the NOT deadenylase complex and for degradation of many mRNAs. Nucleic Acids Res. 41, 1211-1222. doi: 10.1093/nar/gks1133

Funakoshi, Y., Doi, Y., Hosoda, N., Uchida, N., Osawa, M., Shimada, I., et al. (2007). Mechanism of mRNA deadenylation: evidence for a molecular interplay between translation termination factor eRF3 and mRNA deadenylases. Genes Dev. 21 3135-3148. doi: 10.1101/gad.1597707

Garapaty, S., Mahajan, M. A., and Samuels, H. H. (2008). Components of the CCR4NOT complex function as nuclear hormone receptor coactivators via association with the NRC-interacting Factor NIF-1. J. Biol. Chem. 283, 6806-6816. doi: 10.1074/jbc.M706986200

Garces, R. G., Gillon, W., and Pai, E. F. (2007). Atomic model of human Rcd-1 reveals an armadillo-like-repeat protein with in vitro nucleic acid binding properties. Protein Sci. 16, 176-188. doi: 10.1110/ps.062600507

Godwin, A. R., Kojima, S., Green, C. B., and Wilusz, J. (2013). Kiss your tail goodbye: the role of PARN, Nocturnin, and Angel deadenylases in mRNA biology. Biochim. Biophys. Acta 1829, 571-579. doi: 10.1016/j.bbagrm

Goldstrohm, A. C., Hook, B. A., Seay, D. J., and Wickens, M. (2006). PUF proteins bind Pop2p to regulate messenger RNAs. Nat. Struct. Mol. Biol. 13, 533-539. doi: $10.1038 /$ nsmb 1100
Goldstrohm, A. C., Seay, D. J., Hook, B. A., and Wickens, M. (2007). PUF proteinmediated deadenylation is catalyzed by Ccr4p. J. Biol. Chem. 282, 109-114. doi: 10.1074/jbc.M609413200

Goldstrohm, A. C., and Wickens, M. (2008). Multifunctional deadenylase complexes diversify mRNA control. Nat. Rev. Mol. Cell Biol. 9, 337-344. doi: $10.1038 / \mathrm{nrm} 2370$

Gregory, R. C., Lord, K. A., Panek, L. B., Gaines, P., Dillon, S. B., and Wojchowski, D. M. (2000). Subtraction cloning and initial characterization of novel epoimmediate response genes. Cytokine 12, 845-857. doi: 10.1006/cyto.2000.0686

Gutierrez-Camino, A., Lopez-Lopez, E., Martin-Guerrero, I., Piñan, M. A. Garcia-Miguel, P., Sanchez-Toledo, J., et al. (2014). Noncoding RNA-related polymorphisms in pediatric acute lymphoblastic leukemia susceptibility. Pediatr. Res. 75, 767-773. doi: 10.1038/pr.2014.43

Haas, M., Siegert, M., Schürmann, A., Sodeik, B., and Wolfes, H. (2004). c-Myb protein interacts with Rcd-1, a component of the CCR4 transcription mediator complex. Biochemistry 43, 8152-8159. doi: 10.1021/bi035857y

Halter, D., Collart, M. A., and Panasenko, O. O. (2014). The Not4 E3 ligase and CCR4 deadenylase play distinct roles in protein quality control. PLoS ONE 9:e86218. doi: 10.1371/journal.pone.0086218

Hämmerle, M., Gutschner, T., Uckelmann, H., Ozgur, S., Fiskin, E., Gross, M., et al. (2013). Posttranscriptional destabilization of the liver-specific long noncoding RNA HULC by the IGF2 mRNA-binding protein 1 (IGF2BP1). Hepatology 58, 1703-1712. doi: 10.1002/hep.26537

Hiroi, N., Ito, T., Yamamoto, H., Ochiya, T., Jinno, S., and Okayama, H. (2002) Mammalian Rcdl is a novel transcriptional cofactor that mediates retinoic acidinduced cell differentiation. EMBO J. 21, 5235-5244. doi: 10.1093/emboj/cdf521 Horiuchi, M., Takeuchi, K., Noda, N., Muroya, N., Suzuki, T., Nakamura, T., et al. (2009). Structural basis for the antiproliferative activity of the Tob-hCafl complex. J. Biol. Chem. 284, 13244-13255. doi: 10.1074/jbc.M809250200

Hosoda, N., Funakoshi, Y., Hirasawa, M., Yamagishi, R., Asano, Y., Miyagawa, R., et al. (2011). Anti-proliferative protein Tob negatively regulates CPEB3 target by recruiting Cafl deadenylase. EMBO J. 30, 1311-1323. doi: 10.1038/emboj. 2011.37

Hu, G., Kim, J., Xu, Q., Leng, Y., Orkin, S. H., and Elledge, S. J. (2009). A genome-wide RNAi screen identifies a new transcriptional module required for self-renewal. Genes Dev. 23, 837-848. doi: 10.1101/gad.1769609

Ito, K., Takahashi, A., Morita, M., Suzuki, T., and Yamamoto, T. (2011a). The role of the CNOT1 subunit of the CCR4-NOT complex in mRNA deadenylation and cell viability. Protein Cell 2, 755-763. doi: 10.1007/s13238-011-1092-4

Ito, K., Inoue, T., Yokoyama, K., Morita, M., Suzuki, T., and Yamamoto, T. (2011b). CNOT2 depletion disrupts and inhibits the CCR4-NOT deadenylase complex and induces apoptotic cell death. Genes Cells 16, 368-379. doi: 10.1111/j.13652443.2011.01492.x

Jayne, S., Zwartjes, C. G., van Schaik, F. M., and Timmers, H. T. (2006). Involvement of the SMRT/NCoR-HDAC3 complex in transcriptional repression by the CNOT2 subunit of the human Ccr4-Not complex. Biochem. J. 398, 461-467. doi: 10.1042/BJ20060406

Joly, W., Chartier, A., Rojas-Rios, P., Busseau, I., and Simonelig, M. (2013). The CCR4 deadenylase acts with Nanos and Pumilio in the fine-tuning of Mei-P26 expression to promote germline stem cell self-renewal. Stem Cell Reports 1, 411424. doi: 10.1016/j.stemcr.2013.09.007

Kerr, S. C., Azzouz, N., Fuchs, S. M., Collart, M. A., Strahl, B. D., Corbett, A. H., et al. (2011). The Ccr4-Not complex interacts with the mRNA export machinery. PLoS ONE 6:e18302. doi: 10.1371/journal.pone.0018302

Kim, I., Kwak, H., Lee, H. K., Hyun, S., and Jeong, S. (2012). $\beta$-Catenin recognizes a specific RNA motif in the cyclooxygenase-2 mRNA $3^{\prime}$-UTR and interacts with HuR in colon cancer cells. Nucleic Acids Res. 40, 6863-6872. doi: $10.1093 /$ nar/gks331

Lau, N. C., Kolkman, A., van Schaik, F. M., Mulder, K. W., Pijnappel, W. W., Heck, A. J., et al. (2009). Human Ccr4-Not complexes contain variable deadenylase subunits. Biochem. J. 422, 443-453. doi: 10.1042/BJ20090500

Lee, T. I., Wyrick, J. J., Koh, S. S., Jennings, E. G., Gadbois, E. L., and Young, R. A. (1998). Interplay of positive and negative regulators in transcription initiation by RNA polymerase II holoenzyme. Mol. Cell. Biol. 18, 4455-4462.

Mahajan, M. A., Murray, A., and Samuels, H. H. (2002). NRC-interacting factor 1 is a novel cotransducer that interacts with and regulates the activity of the nuclear hormone receptor coactivator NRC. Mol. Cell. Biol. 22, 6883-6894. doi: 10.1128/MCB.22.19.6883-6894.2002 
Maillet, L., Tu, C., Hong, Y. K., Shuster, E. O., and Collart, M. A. (2000). The essential function of Notl lies within the Ccr4-Not complex. J. Mol. Biol. 303, 131-143. doi: 10.1006/jmbi.2000.4131

Maragozidis, P., Karangeli, M., Labrou, M., Dimoulou, G., Papaspyrou, K., Salataj, E., et al. (2012). Alterations of deadenylase expression in acute leukemias: evidence for poly(a)-specific ribonuclease as a potential biomarker. Acta Haematol 128, 39-46. doi: 10.1159/000337418

Mathys, H., Basquin, J., Ozgur, S., Czarnocki-Cieciura, M., Bonneau, F., Aartse, A., et al. (2014). Structural and biochemical insights to the role of the CCR4-NOT complex and DDX6 ATPase in microRNA repression. Mol. Cell 54, 751-765. doi: 10.1016/j.molcel.2014.03.036

Mauxion, F., Faux, C., and Séraphin, B. (2008). The BTG2 protein is a general activator of mRNA deadenylation. EMBO J.27, 1039-1048. doi: 10.1038/emboj.2008.43

Mauxion, F., Prève, B., and Séraphin, B. (2013). C2ORF29/CNOT11 and CNOT10 form a new module of the CCR4-NOT complex. RNA Biol. 10, 267-276. doi: 10.4161/rna.23065

Mittal, S., Aslam, A., Doidge, R., Medica, R., and Winkler, G. S. (2011). The Ccr4a (CNOT6) and Ccr4b (CNOT6L) deadenylase subunits of the human Ccr4-Not complex contribute to the prevention of cell death and senescence. Mol. Biol. Cell 22, 748-758. doi: 10.1091/mbc.E10-11-0898

Morita, M., Oike, Y., Nagashima, T., Kadomatsu, T., Tabata, M., Suzuki, T., et al. (2011). Obesity resistance and increased hepatic expression of catabolismrelated mRNAs in Cnot3+/- mice. EMBO J. 30, 4678-4691. doi: 10.1038/emboj. 2011.320

Morita, M., Suzuki, T., Nakamura, T., Yokoyama, K., Miyasaka, T., and Yamamoto, T. (2007). Depletion of mammalian CCR4b deadenylase triggers elevation of the p27Kip1 mRNA level and impairs cell growth. Mol. Cell. Biol. 27, 4980-4990. doi: 10.1128/MCB.02304-06

Morris, J. Z., Hong, A., Lilly, M. A., and Lehmann, R. (2005). Twin, a CCR4 homolog, regulates cyclin poly(A) tail length to permit Drosophila oogenesis. Development 132, 1165-1174. doi: 10.1242/dev.01672

Moura, R., Pontillo, A., D’Adamo, P., Pirastu, N., Campos Coelho, A., and Crovella, S. (2014). Exome analysis of HIV patients submitted to dendritic cells therapeutic vaccine reveals an association of CNOT1 gene with response to the treatment. $J$. Int. AIDS Soc. 17:18938. doi: 10.7448/IAS.17.1.18938

Mulder, K. W., Brenkman, A. B., Inagaki, A., van den Broek, N. J., and Timmers, H. T. (2007). Regulation of histone H3K4 tri-methylation and PAF complex recruitment by the Ccr4-Not complex. Nucleic Acids Res. 35, 2428-2439. doi: 10.1093/nar/gkm175

Mulder, K. W., Winkler, G. S., and Timmers, H. T. (2005). DNA damage and replication stress induced transcription of RNR genes is dependent on the Ccr4Not complex. Nucleic Acids Res. 33, 6384-6392. doi: 10.1093/nar/gki938

Nakamura, T., Yao, R., Ogawa, T., Suzuki, T., Ito, C., Tsunekawa, N., et al. (2004). Oligo-astheno-teratozoospermia in mice lacking Cnot7, a regulator of retinoid X receptor beta. Nat. Genet. 36, 528-533. doi: 10.1038/ng1344

Neely, G. G., Kuba, K., Cammarato, A., Isobe, K., Amann, S., Zhang, L., et al. (2010) A global in vivo Drosophila RNAi screen identifies NOT3 as a conserved regulator of heart function. Cell 141, 142-153. doi: 10.1016/j.cell.2010.02.023

Newton-Cheh, C., Eijgelsheim, M., Rice, K. M., de Bakker, P. I., Yin, X., Estrada, K., et al. (2009). Common variants at ten loci influence QT interval duration in the QTGEN study. Nat. Genet. 41, 399-406. doi: 10.1038/ng.364

Nousch, M., Techritz, N., Hampel, D., Millonigg, S., and Eckmann, C. R. (2013). The Ccr4-Not deadenylase complex constitutes the main poly(A) removal activity in C. elegans. J. Cell Sci. 126, 4274-4285. doi: 10.1242/jcs.132936

Ogami, K., Hosoda, N., Funakoshi, Y., and Hoshino, S. (2014). Antiproliferative protein Tob directly regulates c-myc proto-oncogene expression through cytoplasmic polyadenylation element-binding protein CPEB. Oncogene 33, 55-64 doi: 10.1038/onc.2012.548

Okazaki, N., Okazaki, K., Watanabe, Y., Kato-Hayashi, M., Yamamoto, M., and Okayama, H. (1998). Novel factor highly conserved among eukaryotes controls sexual development in fission yeast. Mol. Cell. Biol. 18, 887-895.

Panepinto, J. C., Heinz, E., and Traven, A. (2013). The cellular roles of Ccr4-NOT in model and pathogenic fungi-implications for fungal virulence. Front. Genet. 4:302. doi: 10.3389/fgene.2013.00302

Parker, R., and Sheth, U. (2007). P bodies and the control of mRNA translation and degradation. Mol. Cell 25, 635-646. doi: 10.1016/j.molcel.2007.02.011

Paul, P., van den Hoorn, T., Jongsma, M. L., Bakker, M. J., Hengeveld, R., Janssen, L., et al. (2011). A genome-wide multidimensional RNAi screen reveals pathways controlling MHC class II antigen presentation. Cell 145, 268-283. doi: 10.1016/j.cell.2011.03.023

Petit, A. P., Wohlbold, L., Bawankar, P., Huntzinger, E., Schmidt, S., Izaurralde, E., et al. (2012). The structural basis for the interaction between the CAF1 nuclease and the NOT1 scaffold of the human CCR4-NOT deadenylase complex. Nucleic Acids Res. 40, 11058-11072. doi: 10.1093/nar/gks883

Pfeufer, A., Sanna, S., Arking, D. E., Müller, M., Gateva, V., Fuchsberger, C., et al. (2009). Common variants at ten loci modulate the QT interval duration in the QTSCD Study. Nat. Genet. 41, 407-414. doi: 10.1038/ng.362

Poulsen, J. B., Andersen, K. R., Kjær, K. H., Durand, F., Faou, P., Vestergaard, A. L., et al. (2011). Human 2' -phosphodiesterase localizes to the mitochondrial matrix with a putative function in mitochondrial RNA turnover. Nucleic Acids Res. 39, 3754-3770. doi: 10.1093/nar/gkq1282

Sakai, A., Chibazakura, T., Shimizu, Y., and Hishinuma, F. (1992). Molecular analysis of POP2 gene, a gene required for glucose-derepression of gene expression in Saccharomyces cerevisiae. Nucleic Acids Res. 20, 6227-6233. doi: 10.1093/nar/20.23.6227

Sandler, H., Kreth, J., Timmers, H. T., and Stoecklin, G. (2011). Not1 mediates recruitment of the deadenylase Caf1 to mRNAs targeted for degradation by tristetraprolin. Nucleic Acids Res. 39, 4373-4386. doi: 10.1093/nar/gkr011

Schwede, A., Ellis, L., Luther, J., Carrington, M., Stoecklin, G., and Clayton, C. (2008). A role for Cafl in mRNA deadenylation and decay in trypanosomes and human cells. Nucleic Acids Res. 36, 3374-3388. doi: 10.1093/nar/gkn 108

Seiden-Long, I. M., Brown, K. R., Shih, W., Wigle, D. A., Radulovich, N., Jurisica, I., et al. (2006). Transcriptional targets of hepatocyte growth factor signaling and Ki-ras oncogene activation in colorectal cancer. Oncogene 25, 91-102. doi: 10.1038/sj.onc. 1209005

Semotok, J. L., Cooperstock, R. L., Pinder, B. D., Vari, H. K., Lipshitz, H. D., and Smibert, C. A. (2005). Smaug recruits the CCR4/POP2/NOT deadenylase complex to trigger maternal transcript localization in the early Drosophila embryo. Curr. Biol. 15, 284-294. doi: 10.1016/j.cub.2005.01.048

Solana, J., Gamberi, C., Mihaylova, Y., Grosswendt, S., Chen, C., Lasko, P., et al. (2013). The CCR4-NOT complex mediates deadenylation and degradation of stem cell mRNAs and promotes planarian stem cell differentiation. PLoS Genet. 9:e1004003. doi: 10.1371/journal.pgen.1004003

Suzuki, A., Igarashi, K., Aisaki, K., Kanno, J., and Saga, Y. (2010). NANOS2 interacts with the CCR4-NOT deadenylation complex and leads to suppression of specific RNAs. Proc. Natl. Acad. Sci. U.S.A. 107, 3594-3599. doi: 10.1073/pnas.0908664107

Suzuki, A., Saba, R., Miyoshi, K., Morita, Y., and Saga, Y. (2012). Interaction between NANOS2 and the CCR4-NOT deadenylation complex is essential for male germ cell development in mouse. PLoS ONE 7:e33558. doi: 10.1371/journal.pone.0033558

Takahashi, A., Kikuguchi, C., Morita, M., Shimodaira, T., Tokai-Nishizumi, N., Yokoyama, K., etal. (2012). Involvement of CNOT3 in mitotic progression through inhibition of MAD1 expression. Biochem. Biophys. Res. Commun. 419, 268-273. doi: 10.1016/j.bbrc.2012.02.007

Tay, Y., Kats, L., Salmena, L., Weiss, D., Tan, S. M., Ala, U., et al. (2011). Codingindependent regulation of the tumor suppressor PTEN by competing endogenous mRNAs. Cell 147, 344-357. doi: 10.1016/j.cell.2011.09.029

Temme, C., Zaessinger, S., Meyer, S., Simonelig, M., and Wahle, E. (2004). A complex containing the CCR4 and CAF1 proteins is involved in mRNA deadenylation in Drosophila. EMBO J. 23, 2862-2871. doi: 10.1038/sj.emboj.7600273

Temme, C., Zhang, L., Kremmer, E., Ihling, C., Chartier, A., Sinz, A., et al. (2010). Subunits of the Drosophila CCR4-NOT complex and their roles in mRNA deadenylation. RNA 16, 1356-1370. doi: 10.1261/rna.2145110

Tucker, M., Staples, R. R., Valencia-Sanchez, M. A., Muhlrad, D., and Parker, R. (2002). Ccr4p is the catalytic subunit of a Ccr4p/Pop2p/Notp mRNA deadenylase complex in Saccharomyces cerevisiae. EMBO J. 21, 1427-1436. doi: 10.1093/emboj/21.6.1427

Tucker, M., Valencia-Sanchez, M. A., Staples, R. R., Chen, J., Denis, C. L., and Parker, R. (2001). The transcription factor associated Ccr4 and Caf1 proteins are components of the major cytoplasmic mRNA deadenylase in Saccharomyces cerevisiae. Cell 104, 377-386. doi: 10.1016/S0092-8674(01)00225-2

Van Etten, J., Schagat, T. L., Hrit, J., Weidmann, C. A., Brumbaugh, J., Coon, J. J., et al. (2012). Human Pumilio proteins recruit multiple deadenylases to efficiently repress messenger RNAs. J. Biol. Chem. 287, 36370-36383. doi: 10.1074/jbc.M112.373522 
Venturini, G., Rose, A. M., Shah, A. Z., Bhattacharya, S. S., and Rivolta, C. (2012). CNOT3 is a modifier of PRPF31 mutations in retinitis pigmentosa with incomplete penetrance. PLoS Genet. 8:e1003040. doi: 10.1371/journal.pgen. 1003040

Wang, H., Morita, M., Yang, X., Suzuki, T., Yang, W., Wang, J., et al. (2010). Crystal structure of the human CNOT6L nuclease domain reveals strict poly(A) substrate specificity. EMBO J. 29, 2566-2576. doi: 10.1038/emboj. 2010.152

Washio-Oikawa, K., Nakamura, T., Usui, M., Yoneda, M., Ezura, Y., Ishikawa, I., et al. (2007). Cnot7-null mice exhibit high bone mass phenotype and modulation of BMP actions. J. Bone Miner. Res. 22, 1217-1223. doi: 10.1359/JBMR.0 70411

Watanabe, C., Morita, M., Hayata, T., Nakamoto, T., Kikuguchi, C., Li, X., et al. (2014). Stability of mRNA influences osteoporotic bone mass via CNOT3. Proc. Natl. Acad. Sci. U.S.A. 111, 2692-2697. doi: 10.1073/pnas.1316932111

Wiederhold, K., and Passmore, L. A. (2010). Cytoplasmic deadenylation: regulation of mRNA fate. Biochem. Soc. Trans. 38, 1531-1536. doi: 10.1042/BST0381531

Winkler, G. S. (2010). The mammalian anti-proliferative BTG/Tob protein family. J. Cell. Physiol. 222, 66-72. doi: 10.1002/jcp.21919

Winkler, G. S., and Balacco, D. L. (2013). Heterogeneity and complexity within the nuclease module of the Ccr4-Not complex. Front. Genet. 4:296. doi: 10.3389/fgene.2013.00296

Winkler, G. S., Mulder, K. W., Bardwell, V. J., Kalkhoven, E., and Timmers, H. T. (2006). Human Ccr4-Not complex is a ligand-dependent repressor of nuclear receptor-mediated transcription. EMBO J. 25, 3089-3099. doi: 10.1038/sj.emboj.7601194

Yamashita, A., Chang, T. C., Yamashita, Y., Zhu, W., Zhong, Z., Chen, C. Y., et al. (2005). Concerted action of poly(A) nucleases and decapping enzyme in mammalian mRNA turnover. Nat. Struct. Mol. Biol. 12, 1054-1063. doi: $10.1038 / \mathrm{nsmb} 1016$
Yang, X., Morita, M., Wang, H., Suzuki, T., Yang, W., Luo, Y., et al. (2008). Crystal structures of human BTG2 and mouse TIS21 involved in suppression of CAF1 deadenylase activity. Nucleic Acids Res. 36, 6872-6881. doi: 10.1093/nar/gkn825

Zaessinger, S., Busseau, I., and Simonelig, M. (2006). Oskar allows nanos mRNA translation in Drosophila embryos by preventing its deadenylation by Smaug/CCR4. Development 133, 4573-4583. doi: 10.1242/dev.02649

Zheng, X., Dumitru, R., Lackford, B. L., Freudenberg, J. M., Singh, A. P., Archer, T. K., et al. (2012). Cnot1, Cnot2, and Cnot3 maintain mouse and human ESC identity and inhibit extraembryonic differentiation. Stem Cells 30, 910-22. doi: $10.1002 /$ stem. 1070

Zwartjes, C. G., Jayne, S., van den Berg, D. L., and Timmers, H. T. (2004). Repression of promoter activity by CNOT2, a subunit of the transcription regulatory Ccr4not complex. J. Biol. Chem. 279, 10848-10854. doi: 10.1074/jbc.M311747200

Conflict of Interest Statement: The authors declare that the research was conducted in the absence of any commercial or financial relationships that could be construed as a potential conflict of interest.

Received: 20 June 2014; accepted: 04 August 2014; published online: 21 August 2014. Citation: Shirai Y-T, Suzuki T, Morita M, Takahashi A and Yamamoto T (2014) Multifunctional roles of the mammalian CCR4-NOT complex in physiological phenomena. Front. Genet. 5:286. doi: 10.3389/fgene.2014.00286

This article was submitted to Non-Coding RNA, a section of the journal Frontiers in Genetics.

Copyright (0) 2014 Shirai, Suzuki, Morita, Takahashi and Yamamoto. This is an openaccess article distributed under the terms of the Creative Commons Attribution License (CC BY). The use, distribution or reproduction in other forums is permitted, provided the original author(s) or licensor are credited and that the original publication in this journal is cited, in accordance with accepted academic practice. No use, distribution or reproduction is permitted which does not comply with these terms. 